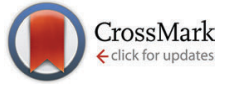

Cite this: Phys. Chem. Chem. Phys., 2014, 16, 23671

\title{
Monophasic ligand-free alloy nanoparticle synthesis determinants during pulsed laser ablation of bulk alloy and consolidated microparticles in water $\dagger$
}

\author{
Anne Neumeister, Jurij Jakobi, Christoph Rehbock, Janine Moysig and \\ Stephan Barcikowski*
}

\begin{abstract}
Chemical syntheses of homogenous solid solution alloy nanoparticles of noble metals require high temperature above $100{ }^{\circ} \mathrm{C}$. Beside this, aqueous co-reduction methods lead to phase separation. In contrast, pulsed laser ablation in liquid (PLAL) allows synthesis of alloy nanoparticles with totally homogeneous ultrastructure in aqueous media at room temperature without reducing agents or organic ligands. However, to date, the dominant alloy formation process during PLAL is not fully understood. Based on the model of $\mathrm{Ag}-\mathrm{Au}$ alloy, we elucidate that the underlying mechanism is not affected by post-irradiation or interactions with colloidal particles in solution but is caused directly by ablation. In this context we analyzed nanoparticles generated from alloy targets with 9 different compositions as well as pure Ag and Au references using UV-Vis spectroscopy, TEM and TEM-EDX line scans. The obtained results highlight that the total composition but not the microstructure of the applied target is the dominant parameter ruling elemental composition in the resulting solid solution alloy nanoparticles. Based on these findings, the application of pressed targets of metal powder mixtures in a continuous laser process with residence time $<60 \mathrm{~s}$ allows economical fabrication of alloy nanoparticles ideally suited for applications in catalysis or biomedicine.
\end{abstract}

Received 25th July 2014,

Accepted 12th September 2014

DOI: $10.1039 / \mathrm{c} 4 \mathrm{cp} 03316 \mathrm{~g}$

www.rsc.org/pccp

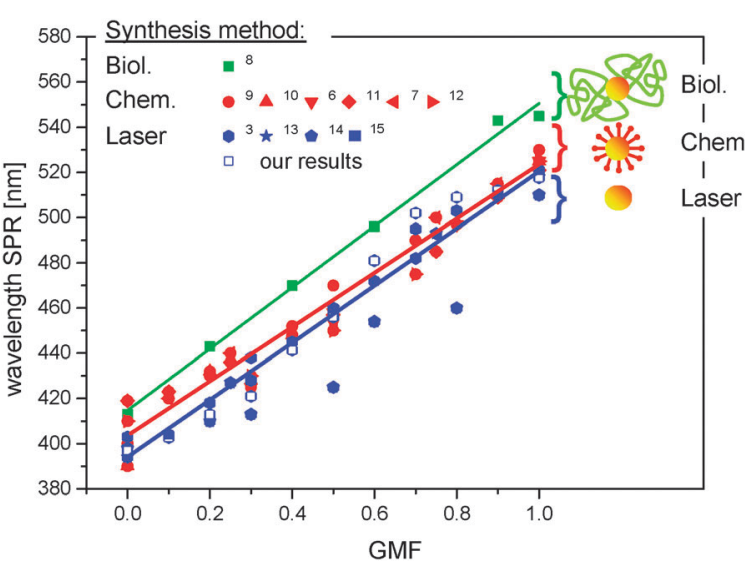

Fig. 1 Surface plasmon resonance peak of $\mathrm{Ag}-\mathrm{Au}$ nanoparticle colloids as a function of gold molar fraction (GMF) and synthesis method. All data derived from biological, chemical and laser-based synthesis methods reveal a linear correlation of the SPR peak maximum with the GMF. Deviating SPR peak positions of NPs from different methods are attributed to surface ligand influences. ${ }^{3-17}$

and laser-based). Overall, the SPR peak position linearly increases with the GMF, whereas recent calculations have emphasized not strongly linear dependency. ${ }^{18}$ Additionally, these data clearly show 
that the SPR peak position depends on the synthesis method that is used to generate the NPs. In contrast to laser-generated alloy NPs, a red-shift of the SPR peak occurs in chemically and biologically synthesized alloy NPs. These differences may be attributed to the fact that during biological and chemical synthesis NPs are invariably stabilized with ligands. The presence of ligands increases the refractive index in the nanovicinity of the particle surface and hence induces a bathochromic shift of the SPR peak maximum, ${ }^{19-21}$ with laser-generated alloy nanoparticles having the lowest SPR wavelengths.

In contrast to the previously mentioned fabrication methods, pulsed laser ablation in liquids (PLAL) enables the generation of ligand-free NPs. ${ }^{22-24}$ This is a prerequisite for a variety of applications, in order to ensure e.g. high catalytic activity, exclusion of toxic effects in biomedical applications or an improved sensitivity in analytical applications. ${ }^{22,25,26}$ Beside these effects, the control of the chemical reaction method for generation of homogeneous $\mathrm{Ag}-\mathrm{Au}$ nanoparticles is difficult due to the different reduction potentials. $^{27}$ Therefore, co-reduction of $\mathrm{Ag}$ and $\mathrm{Au}$ precursors leads to the formation of nanoparticles with a gradient, where more noble metal (Au) is reduced first and is hence more abundant in the core of the formed nanoparticle. ${ }^{28}$

Thus, motivated by the superior nanomaterial properties several studies on laser-based Ag-Au nanoparticle synthesis have been carried out over the last few years. ${ }^{15,17,23,31,32,44}$

The formation of NPs during PLAL entails a number of physicochemical processes which are summarized in Fig. 2. Briefly, the incoming laser pulse is adsorbed by the bulk material forming a plasma (C2). This is followed by the generation of an expanding cavitation bubble, while crystalline NPs are formed inside the cavitation bubble by nucleation and coalescence (C1). ${ }^{29,30}$ Related to materials that escape this confined volume, mechanism B involves ejecting diffusion and interaction of activated species like ions or atom clusters with colloidal NPs present in the surrounding medium. Under stationary conditions, which are generally used for studies of PLAL, the next incoming laser pulse will irradiate the currently generated NPs leading to scattering, absorption, and inciting fragmentation effects (mechanism A).

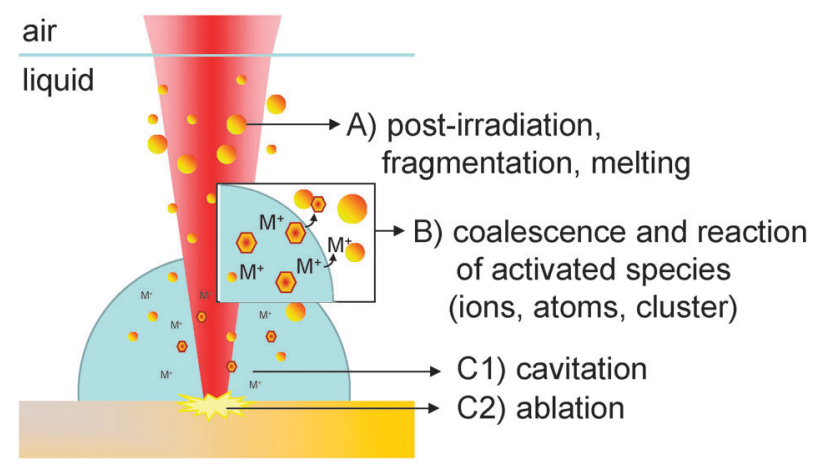

Fig. 2 Mechanisms of nanoparticle synthesis using pulsed laser ablation in liquids with different steps like (a) post-irradiation (interaction of colloidal particles with the laser beam), (b) coalescence and reaction of the generated species with colloidal particles and (c) ablation and cavitation bubble formation.
Accordingly, PLAL-based ligand-free alloy NP synthesis has been successfully conducted based on three methods:

(a) Post-irradiation of $\mathrm{Ag}$ and $\mathrm{Au} \mathrm{NP}$ colloids generated during PLAL ${ }^{3,17,31}$

(b) PLAL of Ag or Au bulk targets in Ag or Au NP colloidal solutions $^{22}$

(c) PLAL of bulk alloy targets ${ }^{15,23,32,44}$

Method (a) is an extensively studied method to fabricate $\mathrm{Ag}$-Au-NPs based on post-irradiation of a colloidal mixture of previously generated Ag- and Au-NPs. This method is sketched as mechanism A of the formerly described processes in Fig. 2. Depending on the pulse length and the laser wavelength, fragmentation of the NPs and subsequent sintering, ${ }^{33}$ melting and diffusion processes are discussed to occur during the formation of bimetallic NPs. ${ }^{34}$ Compagnini et al. showed that re-irradiation first leads to core-shell NPs, later $(t>10 \mathrm{~min})$ succeeded by alloy NP formation. ${ }^{31}$ In accordance, Besner et al. proposed that after colloidal mixtures of $\mathrm{Ag}$ and $\mathrm{Au}$ are postirradiated for 20 minutes by femtosecond white light filaments, $\mathrm{Ag}$ atoms diffuse into Au NPs and homogenous NP alloys are formed. ${ }^{17}$ Another study showed that post-irradiation of a colloidal mixture of $\mathrm{Au}$ and $\mathrm{Ag}$ resulted in alloy NP formation after 25 min. ${ }^{3}$ Furthermore, the bimetallic composition of the nanoparticles is controlled via the initial molar ratio of $\mathrm{Au}$ and $\mathrm{Ag}$ nanoparticles in solution. ${ }^{17,33}$

A second method (b) to generate $\mathrm{Ag}-\mathrm{Au}$ alloy NPs has recently been studied by Intartaglia et al. Thereby, pulsed laser ablation of $\mathrm{Ag}$ or $\mathrm{Au}$ bulk is carried out in a colloidal solution of the other constituent. This experimental approach covers combinations of mechanisms A and B sketched in Fig. 2. During this process, both might occur, an Ag-Au-NP formation directly during ablation of one component due to the presence of NPs of the other constituent (mechanism B of Fig. 2) as well as an alloying afterwards through re-irradiation (mechanism A of Fig. 2). But the experimental design of method (b) inherently makes the differentiation between the dominant mechanisms difficult. The composition of the Ag-Au alloy NPs is tunable by adjusting the concentration of the colloidal solution prior to the irradiation process, the laser wavelength and the irradiation time. ${ }^{22}$

In contrast to (a) and (b), the third synthesis method (c) involves ablation of a bulk target by PLAL in a one-step process. Lee et al. previously demonstrated that Ag-Au-NPs preserve the stoichiometry of the target during $30 \mathrm{~min}$ of PLAL. ${ }^{15}$ At such long processing times, two processes, ablation and post-irradiation, take place and differentiation between them is difficult. However, it is yet unclear at which stage of the formerly described mechanisms A, $\mathrm{B}, \mathrm{C}$ during PLAL the alloying NP formation dominantly occurs. Derived from the three interacting mechanisms, we designed the experimental approach as depicted in Fig. 3 which allows us to clearly differentiate between A-C. In contrast to the literature, we examined the mechanism during shorter ablation or irradiation times in order to simulate quasi single pulse experiments. In addition, the influence of both, the target composition and its microstructure on the NP alloy formation process during PLAL is studied using bulk alloy targets as well as a mixture of $\mathrm{Ag}$ and $\mathrm{Au}$ microparticles pressed as pellet targets. 


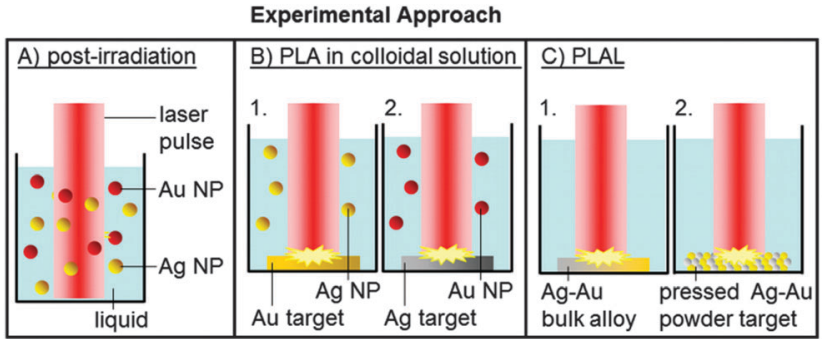

Fig. 3 Experimental approach divided into (A) post-irradiation, (B) pulsed laser ablation in colloidal solution and (C) pulsed laser ablation of (1) bulk alloy and (2) pressed Ag-Au microparticle powder target.

\section{Experimental}

\section{Materials}

$\mathrm{Ag}_{100-x}-\mathrm{Au}_{x}$ alloy bulk ( $x=10$ to 90 mol\% in $10 \%$ intervals) were obtained from Research Institute for Precious Metals and Metals Chemistry (FEM, Germany). For the measured density of alloy targets see ESI $\dagger$ (Fig. S1). The pure silver foil (99.99\%) was purchased from Goodfellow GmbH (Germany) and gold foil (99.99\%) from Allgemeine Gold und Silberscheidenanstalt AG (Germany). Ag micro powder ( $\geq 99.9 \%, 4-7 \mu \mathrm{m})$ and Au micro powder $(\geq 99.9 \%, 5.5-9.0 \mu \mathrm{m})$ were purchased from Alfa Aesar.

The water used for all experiments was purified using a Millipore Ultrapure water system to a resistivity of $18.2 \mathrm{M} \Omega \mathrm{cm}$ at $25{ }^{\circ} \mathrm{C}$.

\section{Methods}

Nanoparticle synthesis was performed using a Nd:YAG ns-laser (Innolas SpitLight DPSS250-100) at a wavelength of $1064 \mathrm{~nm}$ and $532 \mathrm{~nm}$ with a repetition rate of $100 \mathrm{~Hz}$ and a laser fluence of $575 \mathrm{~mJ} \mathrm{~cm} \mathrm{~cm}^{-2}$ (calculated for unfocussed laser beam in ambient gas). During PLAL, targets were placed on the bottom of a glass vessel filled with $5 \mathrm{~mL}$ of water (Fig. 3C) or $\mathrm{Ag}$ and $\mathrm{Au}$ colloidal solution (Fig. 3B). The liquid layer above the target was $20 \mathrm{~mm}$. If not stated otherwise the experiments were carried out at the same fluence without focusing of the beam with a raw beam diameter of $4 \mathrm{~mm}$, using the fundamental wavelength of $1064 \mathrm{~nm}$ and a process time of $60 \mathrm{~s}$. The detected spot size on the target surface after ablation was $1.8 \mathrm{~mm}$ (Fig. S2, ESI $\dagger$ ). The measured particle size from the TEM micrographs for all nanoparticle compositions was around $10 \mathrm{~nm}$ (Fig. S3, ESI $\dagger$ ).

Post-irradiation experiments were performed after mixing $\mathrm{Ag}$ and $\mathrm{Au}$ colloids generated during PLAL in water (Fig. 3A). The molar concentrations of the colloidal Ag and Au NPs corresponded to half of the concentration of NPs in solution achieved during $15 \mathrm{~s}$ of laser ablation of $\mathrm{Au}$ and $\mathrm{Ag}$ bulk targets, respectively.

$\mathrm{Ag}$ and $\mathrm{Au}$ powders at different ratios $\left(\mathrm{Ag}_{x} \mathrm{Au}_{100-x}, x=0,20\right.$, $40,50,60,80 \mathrm{~mol} \%$ ) were mechanically stirred for 30 minutes prior to compression molding. The mixture was pressed with a total pressure of 10 tons $(0.739 \mathrm{GPa})$ for $10 \mathrm{~min}$ to form compact targets based on a procedure previously used by Zhang et al. ${ }^{16}$ All the operations were performed at room temperature.
The targets had a thickness of $0.2 \mathrm{~mm}$ and a diameter of $13 \mathrm{~mm}$. For a digital microscope image of the target surface see $\mathrm{ESI} \dagger$ (Fig. S2).

\section{Characterization}

The optical absorption spectra of the colloids were recorded in a quartz cuvette (Helma Analytics, $10 \mathrm{~mm}$ ) at room temperature using an Evolution $201 \mathrm{UV}$-Vis spectrometer in the range of 300 to $800 \mathrm{~nm}$. The extinction signal of the spectrometer has been calibrated by 667.305-UV standard (Hellma GmbH \& Co. KG).

Transmission electron microscopy (TEM) images were acquired using a Philips CM 12 at an acceleration voltage of $120 \mathrm{kV}$. The TEM-EDX measurements were carried out using a FEI Tecnai F20 transmission electron microscope (acceleration voltage $200 \mathrm{kV}$ ). For sample preparation, a small droplet of freshly prepared colloidal solution was deposited onto a carbon-coated copper grid and air dried. The particle size was determined by measuring the diameter of more than 1000 individually dispersed particles identified in TEM images by using the software ImageJ.

The pressed micropowder targets were characterized by scanning electron microscopy with a Hitachi TM3000. The composition of the target was detected using an energy dispersive X-ray (EDX) spectrometer Quantax 70 from Bruker Nano GmbH.

Ablation rates were determined by weighing the target prior to and after the ablation process using a microbalance (Precisa 40SM-200A, Precisa Gravimetrics AG, Switzerland) with a $10 \mu \mathrm{g}$ accuracy.

\section{Results and discussion}

\section{Results}

Post-irradiation of mixed Ag and Au colloidal solution. Fig. 4 shows the UV-Vis spectra of a colloidal mixture of $\mathrm{Au}$ and $\mathrm{Ag}$ NPs at a 1:1 molar ratio after post-irradiation for up to $90 \mathrm{~s}$. Over the whole irradiation time range, spectra reveal two distinct absorbance maxima located at around 397 and $509 \mathrm{~nm}$, corresponding to $\mathrm{Ag}$ and $\mathrm{Au}$ plasmon bands, respectively. Hence, no alloy formation is spectroscopically detectable for an irradiation time of $90 \mathrm{~s}$. Ag nanoparticles normally show more intense plasmonic features in comparison to $\mathrm{Au}$ nanostructures of similar size

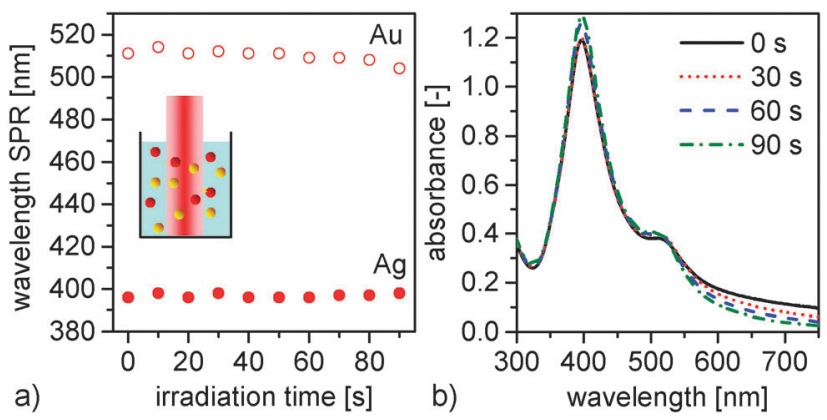

Fig. 4 Post-irradiation of $\mathrm{Au}$ and $\mathrm{Ag}$ colloidal mixtures reveals no alloy formation but isolated particle fractions. (a) Wavelength of surface plasmon resonance of $\mathrm{Ag}$ and $\mathrm{Au}$, respectively, as a function of irradiation time; (b) spectra of $\mathrm{Ag}$ and $\mathrm{Au}$ colloidal solution after different times of post-irradiation. 
and shape. The plasmon band absorption of Ag NPs is narrower and higher compared to plasmon band absorption of Au NPs due to the higher molar extinction coefficient and narrower plasmon band width of Ag NPs. ${ }^{35}$ Even if there would be a formation of $\mathrm{Ag}-\mathrm{Au}$ alloy NPs, this fraction is not dominant and does not contribute to the extinction signal detected in our experiments.

PLA in colloidal solution. At the same fluence of the postirradiation experiment, pulsed laser ablation of targets immersed in colloids has been carried out. Pulsed laser ablation of Au was carried out in a Ag NPs solution. Fig. 5a shows the wavelength of the SPR peak of $\mathrm{Au}$ and $\mathrm{Ag}$ at variable ablation times. As the ablation time increases, the SPR peak position of $\mathrm{Ag}$ remains unchanged while the SPR peak position of Au is slightly shifted towards shorter wavelengths. Within the first $90 \mathrm{~s}$ the SPR peak position of $\mathrm{Au}$ is higher than $500 \mathrm{~nm}$, which indicates that during the first minute of ablation no alloy formation occurs or is detectable. Furthermore, spectra show almost no difference in NPs generated using the fundamental wavelength $(\lambda=1064 \mathrm{~nm})$ in comparison to spectra of NPs fabricated using the second harmonic wavelength $(\lambda=532 \mathrm{~nm})$.

In Fig. $5 \mathrm{~b}$ the ratio between the intensity of $\mathrm{Au}$ and $\mathrm{Ag}$ is depicted and shows a linear incline with increasing ablation time. As expected, the Au NP concentration increases with longer Au target ablation times. No dominant shielding effect of the laser beam due to the presence of Ag NPs in solution is therefore detectable during this short time period.

In the following experimental series target and colloid material were switched. The results of PLAL of Ag in Au NP colloidal solution
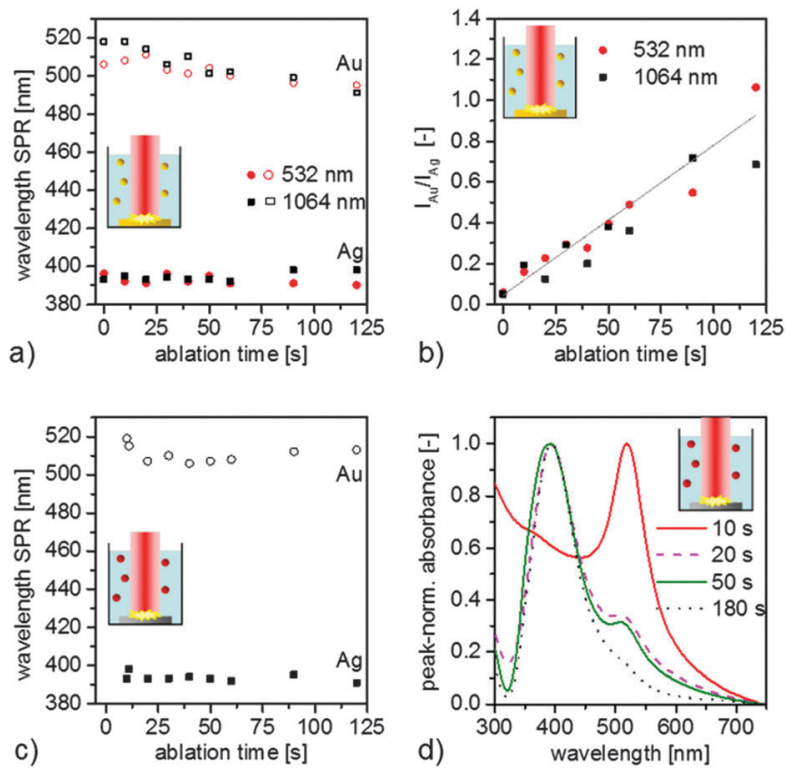

Fig. 5 Nanosecond laser ablation of metal targets in the presence of colloidal nanoparticles ( $a$ and $b$ : Au targets + Ag colloids; $c$ and $\mathrm{d}$ : Ag targets $+\mathrm{Au}$ colloids) shows no significant alloying. (a) Wavelength of SPR peak position of $\mathrm{Ag}$ and Au versus the ablation time and (b) SPR peak intensity of Au in relation to $\mathrm{Ag}$ as a function of ablation time during PLAL (at a laser wavelength of $1064 \mathrm{~nm}$ and $532 \mathrm{~nm}$ ) of Au in Ag NP colloidal solution; (c) wavelength of SPR of $\mathrm{Au}$ and $\mathrm{Ag}$ as a function of ablation time during PLAL of Ag in Au NP colloidal solution; (d) peak-normalized spectra of Ag and Au NP colloidal solution during PLAL of $\mathrm{Ag}$ in Au NP colloidal solution at different ablation times. are demonstrated in Fig. $5 \mathrm{c}$ and d. Fig. 5c shows the wavelength of the SPR peak of $\mathrm{Ag}$ and Au with increasing ablation time of the $\mathrm{Ag}$ target. Up to an ablation time of $120 \mathrm{~s}$ there is no shift of the SPR band of Ag detectable while a slight initial decrease of the SPR band of $\mathrm{Au}$ from $520 \mathrm{~nm}$ to $500 \mathrm{~nm}$ within $10 \mathrm{~s}$ is observed. Exemplary UV-Vis spectra in Fig. $5 \mathrm{~d}$ show that the SPR peak contribution of $\mathrm{Au}$ diminishes continuously in its contribution of the total SPR spectrum with higher ablation times due to increasing concentrations of Ag NPs in solution. No shift of the plasmon peak position and no new SPR peak are observed indicating no formation of alloy NPs under these short irradiation time conditions.

In summary, during the first minute of ablation, no alloy NP formation induced by post-irradiation or interactions with previously released $\mathrm{Au}$ or $\mathrm{Ag} \mathrm{NP}$ is detectable. Hence, an ablation time of $60 \mathrm{~s}$ was used for the following experiments allowing us to exclude potential side-effects from previously investigated mechanisms.

PLAL of bulk alloy targets. Pulsed laser ablation of solid alloy targets in water was carried out with the same laser fluence as mentioned above. Fig. 6 shows the results of PLAL of alloy bulk targets with varying gold and silver molar fraction. The wavelength of the SPR of NPs generated during PLAL is plotted against the GMF in Fig. 6a. The plasmon band is red-shifted
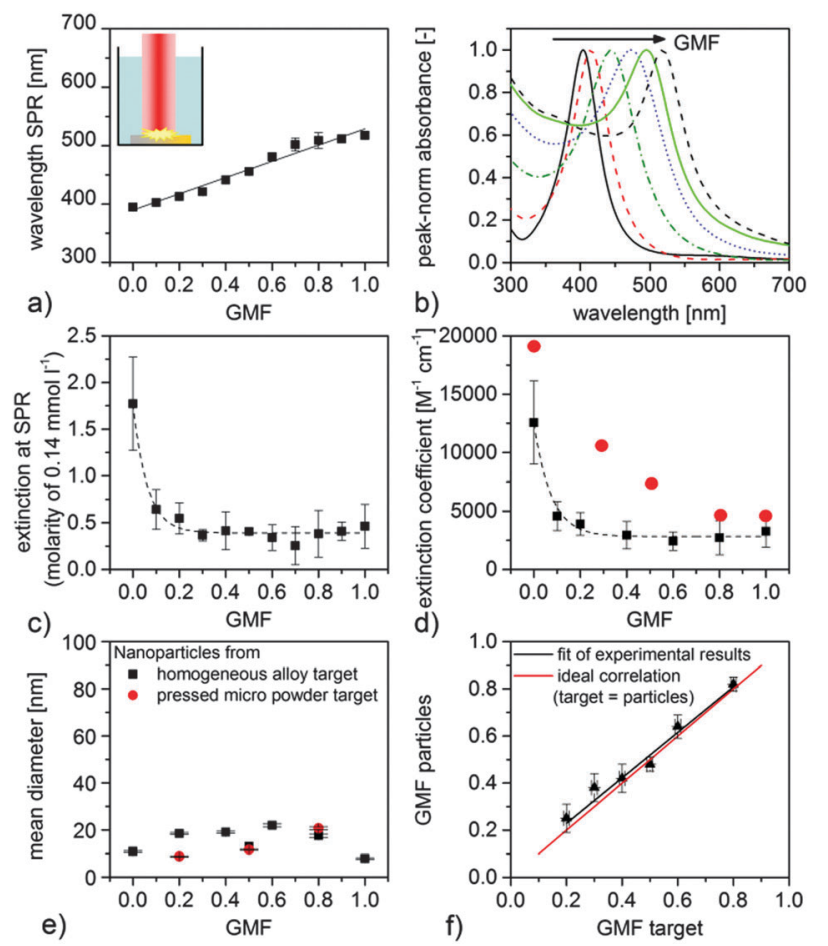

Fig. 6 PLAL of bulk alloy targets in pure water causes homogeneous alloy NP formation. (a) Wavelength of surface plasmon resonance peak of $\mathrm{Ag}-\mathrm{Au}$ nanoparticle colloids generated during $60 \mathrm{~s}$ PLAL of alloy bulk as a function of GMF and (b) peak-normalized UV-Vis spectra of colloids; (c) extinction at SPR at a molarity of $0.14 \mathrm{mmol} \mathrm{L}^{-1}$ and (d) extinction coefficient as a function of GMF. Red circles represent values taken from Link et al. ${ }^{6}$ (e) Number weighted particle size as a function of GMF. These values were taken from the peak maxima of log normal-fitted particle size distributions from TEM images. (f) Correlation of GMF of laser-generated nanoparticles measured by TEM-EDX with GMF of the bulk alloy targets. 
(between $393 \mathrm{~nm}(\mathrm{Ag})$ and $529 \mathrm{~nm}(\mathrm{Au})$ ) with an increasing amount of gold following a linear relationship corresponding to results found in the literature (Fig. 1 and 6a; compare ablation in acetone and methanol in Fig. S4 in ESI $\dagger$ ). Even though slight deviations from linearity were found, they are $<4.2 \%$. This is well within the error range of the experiment (compare error bars in Fig. 6a) and does not constitute a significant trend. Furthermore, it is generally known that the SPR peak position in pure $\mathrm{Au}$ and $\mathrm{Ag}$ nanoparticles is size dependent. ${ }^{36-39}$ However, this factor seems to be negligible in our experiments as the particle size did not significantly change with the GMF (Fig. 6e). Maybe effects attributed to particle composition clearly overshadow possible crystal size effects. Plasmon resonance spectra have been recorded in the colloidal state, so that hydrodynamic phenomena like plasmon coupling caused by agglomeration may also be a contributing factor. ${ }^{18}$ The spectra of the generated NPs, which are depicted in Fig. 6b, show distinct and single peaks clearly indicating formation of homogenous (solid solution) alloy NPs. Two bands would be expected in the case of NPs with a phase segregation or pronounced core-shell structure. ${ }^{40}$

In order to compare the extinction characteristics of ligandfree $\mathrm{Ag}-\mathrm{Au}$ NPs in solution, Fig. 6c shows the extinction at the SPR maximum at an equal molarity of $0.14 \mathrm{mmol} \mathrm{L}^{-1}$. As the GMF increases the extinction of the SPR peak maximum decreases exponentially. In the same manner the extinction coefficient (integrated over the whole UV-Vis spectrum) decreases with increasing GMF, depicted in Fig. 6d. The extinction coefficient is found to be almost constant for GMF $>0.4$.

In this diagram, data of extinction coefficients reported by Link et al. are added for comparison which are in good agreement with our results. ${ }^{6}$ For instance, an extinction coefficient for
Au NPs of $4400 \mathrm{M}^{-1} \mathrm{~cm}^{-1}$ derived from our study is comparable with $4000 \mathrm{M}^{-1} \mathrm{~cm}^{-1}$ taken from the literature. The extinction coefficient of GMF below 0.5 measured by Link et al. is higher which might be due to differences in the sizes of the generated NPs since the extinction coefficient is strongly size-dependent. Correspondingly, an exponential relationship is found between the extinction coefficient and GMF in the literature and our data consistently.

To validate the UV-Vis measurements of the colloids indicating monophasic alloy nanoparticles, analysis of the intra-particle element distribution and nanoparticle composition was carried out by TEM-EDX analysis. The representative line scans and selected-area composition measurements of nanoparticles are shown together with associated UV-Vis spectra of the colloids in Fig. 7. According to the line scans, both elements are homogenously distributed in the analysed nanoparticles. Formation of phase boundaries or segregation of the elements, which is well detectable with this technique as recently shown by Malviya et al., ${ }^{41}$ was not detected. To verify these results, the line scan analysis was done on ten differently sized nanoparticles for each GMF (see Fig. S5 in ESI $\dagger$ ). Nevertheless, the EDX analysis of selected nanoparticles indicates that their composition slightly deviates from the bulk alloy material (Fig. 6f) at low GMF. For these silver-rich compositions, the resulting NPs contain less silver than their bulk counterpart. This behaviour of silver-based nanoparticles in aqueous solutions is well known and could be explained by $\mathrm{Ag}$ oxidation and $\mathrm{Ag}^{+}$ion release..$^{42-44}$ In our case, deviation of the silver content for GMF 0.2 is 5 at\% which corresponds to silver depletion up

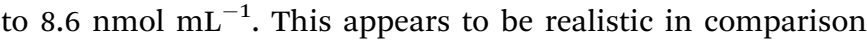
to the ion release of citrate stabilized silver nanoparticles produced by chemical synthesis. ${ }^{45}$
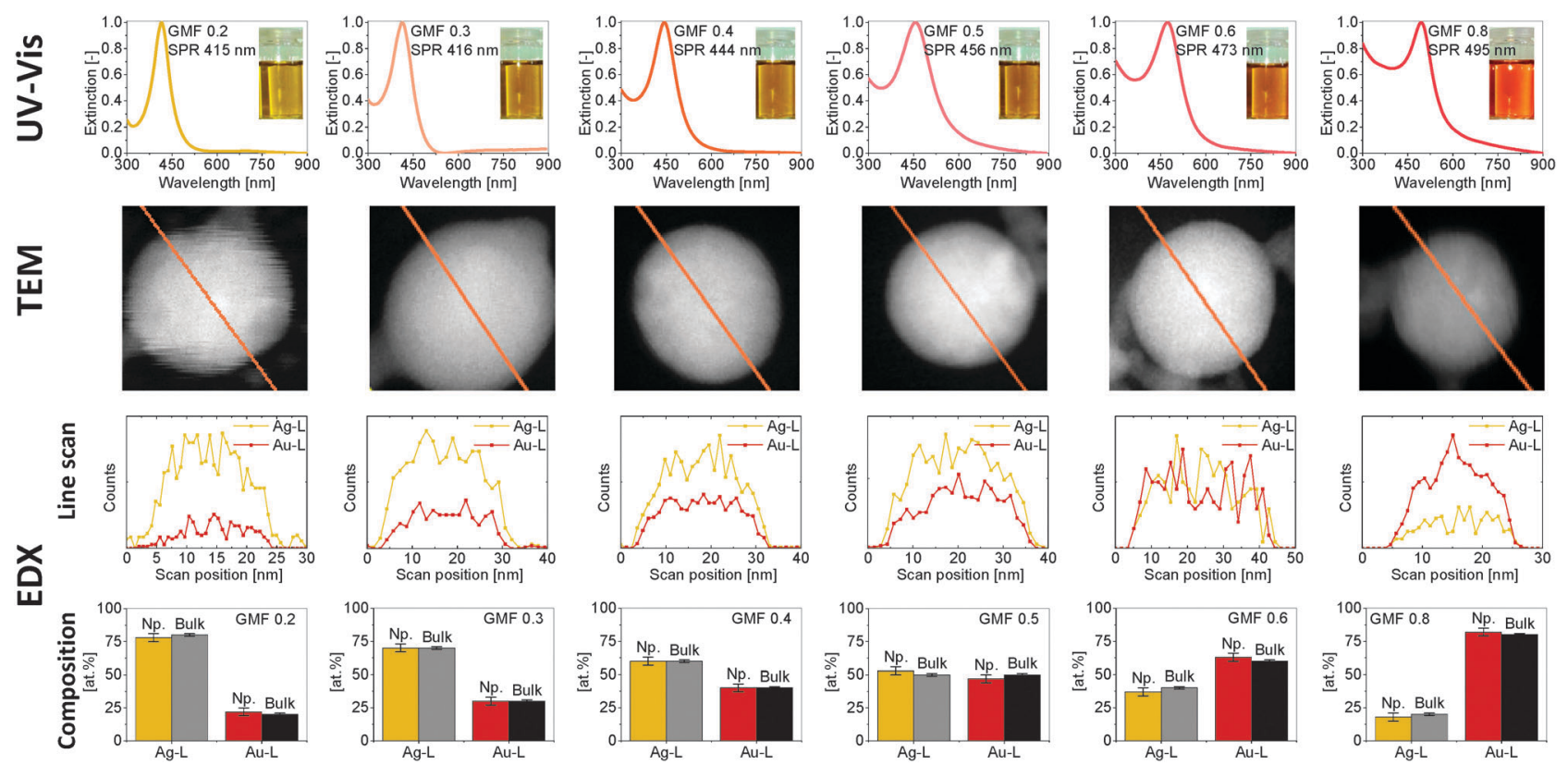

Fig. 7 Comparison of UV-Vis spectra of Ag-Au colloids, element distribution (TEM-EDX line scans) and composition of single Ag-Au nanoparticles generated during PLAL of bulk alloys with different GMF (EDX). The composition information of the bulk target is according to the X-ray fluorescence analysis. The analysis confirms formation of homogeneous and monophasic alloy nanoparticles with composition similar to ablated bulk target (see also 120 line scans in Fig. S5, ESI $†$ ). 
In summary, it is evident that for the $\mathrm{Ag}-\mathrm{Au}$ system aqueous synthesis of monophasic nanoparticles with homogenously distributed elements is possible over the whole molar fraction range.

In general, it is evident that in contrast to former results during experimental approaches A and B, a process time of $60 \mathrm{~s}$ is enough to form alloy NPs from alloy bulk during PLAL. Consequently, the alloy formation does not require interaction with dispersed colloids but is directly caused by the laser impact on the bulk material. In order to further elucidate the influence of the target material on alloy formation during PLAL we switched from a homogeneously distributed bulk alloy target to a pressed powder target consisting of micrometer-sized particles.

PLAL of pressed micro powder mixture targets. Laser ablation of pressed $\mathrm{Ag}$ and $\mathrm{Au}$ micro powder target with different amounts of $\mathrm{Au}$ has been carried out in deionised water. Results of the wavelength of the SPR as a function of GMF together with results from PLAL of homogenous alloy bulk are shown in Fig. 8. A good agreement could be found between NPs generated during PLAL of pressed micro powder and NPs generated during PLAL of alloy bulk targets. Both targets allow fabrication of alloy NPs (Fig. 8a). In order to explain these results, a detailed analysis of the composition of the pressed micro powder target was conducted.

Fig. 8b shows the EDX mapping of the pressed micro powder target cross section. The inner structure of the target consists of single spherical particles with distinct areas of $\mathrm{Ag}$ and $\mathrm{Au}$ particle assemblies. It is shown that the target is bimetallic and no solid-solution alloy was present. $\mathrm{Ag}$ and $\mathrm{Au}$ microparticles are distributed randomly inside the target. Microparticles as a dry powder are often agglomerated and mixing is frequently not sufficient to completely separate all single particles. Since the diameter of the laser spot on the target is $1.8 \mathrm{~mm}$ (Fig. S2, ESI $\dagger$ ) and the average particle size obtained from SEM images (Fig. 8c) of the $\mathrm{Ag}$ and $\mathrm{Au}$ microparticles is $3 \mu \mathrm{m}$, it results in the irradiation of more than $0.36 \times 10^{6}$ consolidated microparticles per pulse. Thus, the laser beam irradiates an area big enough to ensure an ablation of $\mathrm{Ag}$ and $\mathrm{Au}$ microparticle fractions consistent with the ratio of
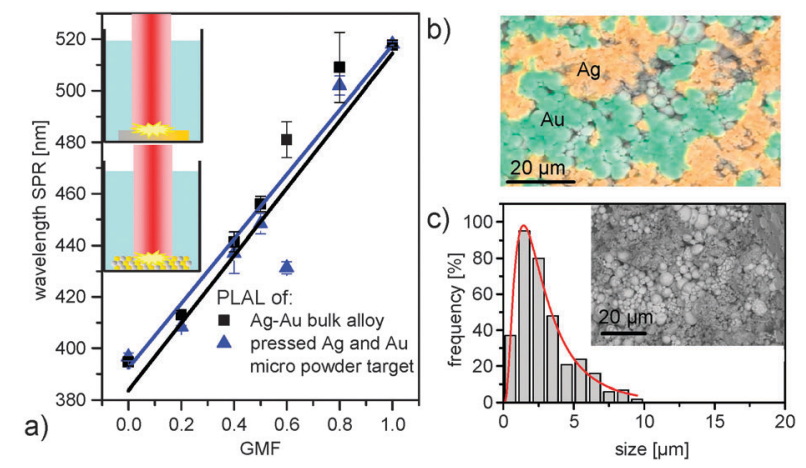

Fig. 8 Comparison of SPR band position of $\mathrm{Ag}-\mathrm{Au}$ colloids produced with PLAL from bulk alloy and pressed Ag and Au micro powder target (a), EDX mapping of pressed Ag-Au micro powder target cross section (b), microparticle size distribution and SEM image (c) of pressed $\mathrm{Ag}$ and $\mathrm{Au}$ micro powder target (GMF 0.4).
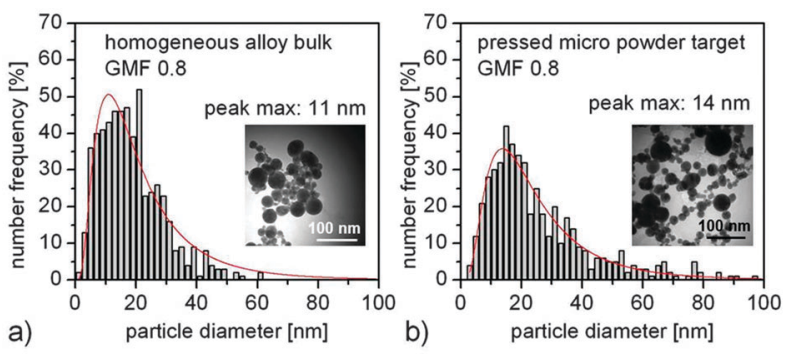

Fig. 9 Representative TEM images and size distribution of NPs generated during PLAL of homogeneous alloy bulk (a) and of pressed micro powder target (b) with a GMF of 0.8 .

$\mathrm{Ag}$ and $\mathrm{Au}$ fractions mixed prior to target fabrication. The size distribution of the pressed microparticles shows slightly smaller particles compared to the specifications of the manufacturer (5.5-9 $\mu \mathrm{m}$ for gold and 4-7 $\mu \mathrm{m}$ for silver powder) probably due to the different size measurement methods.

Exemplarily determined size distributions of generated NPs are depicted in Fig. 9 and show a relatively broad distribution for NPs generated during ablation of bulk alloy target (polydispersity index (PDI) 0.9) as well as of NPs generated during ablation of the pressed micro powder target (PDI 0.8). The curves were fitted with a lognormal function and peak maxima of alloy bulk $(11 \mathrm{~nm})$ and pressed targets $(14 \mathrm{~nm})$ are in a comparable regime. The inset showing representative TEM micrographs confirms the presence of spherical primary nanoparticles in both cases.

\section{Discussion}

During the post-irradiation experiments of $\mathrm{Au}$ and $\mathrm{Ag}$ colloids in solution, short irradiation times $(<90 \mathrm{~s})$ revealed no detectable formation of alloy NPs. It cannot be excluded that a small fraction of alloy NPs are formed during nanosecond-pulsed laser irradiation but not dominant enough to be above the detection limit of the spectrometer. During post-irradiation, the colloidal monometallic NPs absorb the laser pulse. If the absorbed energy is high enough, Ag and Au NPs can be heated above their melting and boiling points inducing a reaction of the NPs with other NPs, which is a concentration and time controlled process. ${ }^{22,34}$ Thus, post-irradiation methods used to successfully fabricate homogenous Ag-Au alloy NPs were found to require relatively long irradiation times (more than $10 \mathrm{~min}$ ) and/or high intensity pulses like femtosecond filaments found in literature. ${ }^{3,17,31}$ But in our case, nanosecond laser pulses and short processing time conditions were chosen. In the determination of the dominant factor in the alloy formation process during PLAL, post-irradiation (mechanism A, in Fig. 2) might therefore be excluded.

Furthermore, during PLA in colloidal solution no alloy formation is detectable below an ablation time of $120 \mathrm{~s}$. This experimental approach was chosen in order to examine whether activated species or atoms ejected during ablation of $\mathrm{Ag}$ or $\mathrm{Au}$, respectively, might interact with the NPs of the other 
component already present in solution besides or in combination with post-irradiation effects. Using a similar experimental approach, Intartaglia et al. fabricated alloy NPs after longer ablation times of $10 \mathrm{~min}$. This seems to indicate that during this alloying process post-irradiation, high extinction cross sections of the particles in order to absorb laser energy and ultrashort laser pulse duration are necessary prerequisite. ${ }^{22}$ This was further backed by examinations on the laser wavelength dependency. In contrast to Intartaglia et al., we found no wavelength dependency indicating that absorption effects during short ablation times are not significant for alloying, at least in the nanosecond pulsed laser regime. Furthermore, alloying by collision of activated nanoparticle species is also meant to be a concentration dependent process which can be neglected due to the short time scale used in our experimental design. As alloying by post-irradiation and collision of activated nanoparticles can be excluded during nanosecond-laser, short-interval processing, we suggest that the dominant alloy formation is directly caused by ablation of the target (Fig. 2, mechanism C).

This third experimental approach revealed that only $60 \mathrm{~s}$ of ablation of $\mathrm{Ag}-\mathrm{Au}$ alloy bulk is sufficient to dominantly generate $\mathrm{Ag}-\mathrm{Au}$ NPs with characteristic plasmon absorption peaks and composition closely resembling the bulk target. This confirms that the alloy formation is identified to occur directly by ablation (Fig. 2, mechanism $\mathrm{C}$ ).

Our results are consistent with the explanation for the alloyed particle formation given by Lee et al. who used 30 min to generate alloy NPs during PLAL of alloy bulk. ${ }^{15}$ The authors of that study proposed that immediately after the laser irradiation, atoms and/or small particles would be ejected through vaporization, followed by the build-up of a dense cloud of metal atoms ( $\mathrm{Ag}$ and $\mathrm{Au}$ atoms) near the laser spot. Our last experimental approach of changing the constitution of the target from bulk alloy to a pressed mixture of $\mathrm{Ag}$ and $\mathrm{Au}$ microparticles showed almost no difference in the formation and composition of alloy NPs. Hence, the formation of nanoparticles and clusters is independent from the microstructure of in the ablated target, at least for structures significantly smaller than the laser spot diameter. The ablated spot of pressed micro powder target in our experiments shows laterally separated $\mathrm{Ag}$ and $\mathrm{Au}$ microparticles. Despite of this, homogeneous alloy nanoparticles are found, which points at sufficient mixing in plasma plume or cavitation bubble. A slight difference in the size of the NPs fabricated from the alloy and the pressed target has been determined which might be due to differences in the target's surface grain structure, which occurs during mixing of the element atoms. It has been recently demonstrated that the grain size of the target influences the size of the NP generated during laser ablation. $^{46}$

It cannot be exactly determined if the formation happens during laser-induced cavitation (Fig. 2, C1) or already in the plasma plume (Fig. 2, C2) since the analytics applied in our experiments is not sufficiently sensitive to discriminate between these processes occurring on a very short time scale. It is clear that inside the cavitation bubble crystalline NPs are already formed and present during PLAL. ${ }^{29,30}$ However, the exact time point of solid solution formation of the $\mathrm{Ag}$ and $\mathrm{Au}$ species cannot be elucidated.

Finally, it can be concluded that the alloy nanoparticle formation process during ablation of alloy targets is fundamentally different from the one occurring based on post-irradiation, fragmentation, and melting. The main discriminator in this case is the required irradiation time, which is much shorter for NP generation during ablation based on an explosive rearrangement of atoms in the cavitation bubble. Positively speaking, it seems that nanosecond laser ablation is an appropriate method to fabricate monophasic aqueous alloy nanoparticles, without disturbance by post-irradiation effects.

\section{Conclusion}

The obtained results seem to indicate that alloy formation during PLAL is found to happen either directly in the plasma plume or inside the cavitation bubble during coalescence and growth of the particles. This is backed by findings showing no detectable side-effects due to post-irradiation at $60 \mathrm{~s}$ or colloidal nanoparticles in solution. In order to fabricate ligand-free alloy NPs with tunable compositions in a one-step process, we recommend the use of PLAL carried out in a liquid flow chamber with a residence time of $\leq 60 \mathrm{~s}$. A mass yield of, e.g. $\mathrm{AgAu}(50: 50)$ NPs of $840 \mathrm{mg}$ per day is achievable at a laser power of $14 \mathrm{~W}$ (see data of ablation rates in Fig. S6 in ESI $\dagger$ ). Assuming a continuous flow rate of $10 \mathrm{~mL} \mathrm{~min}{ }^{-1}$, the permanent outflow concentration of ligand-free alloy NPs would be $175 \mathrm{mg} \mathrm{L}^{-1}$.

Fabrication of Ag-Au alloy NPs is successful by PLAL of either bulk alloy or pressed micro powder target composed of a mixture of both elements. This indicated that the microsegregation of the target is mixed by the ablation process, either in the early plasma or liquid spallation phase, or later during the cavitation in the liquid confinement. Since $\mathrm{Ag}-\mathrm{Au}$ bulk alloys with tunable compositions are difficult to obtain, the generation of alloy NPs during PLAL of pressed micro powder target composed of a mixture of both elements is a smart alternative method simple to fabricate. In these consolidated targets, the composition of the later nanoparticle crystals can be easily tuned by weighing the micropowder fractions before mixing. Interestingly, even though the alloy constituents are segregated on micro scale, mixing of the elements is caused by laser ablation in liquid confinement. This allows the elemental atoms to resolidify in the monophasic solid solution state, homogeneous on the nanoscale. This method may be transferable to other alloys found to be interesting for applications such as optics, ${ }^{23,47}$ catalysis $^{16,48}$ or biomedicine. ${ }^{32,44}$

\section{Acknowledgements}

The authors thank the "Deutsche Forschungsgemeinschaft (DFG)" for funding the project BA 3580/RA 989 and the priority program 1313. We also thank Ms Selina van der Meer for the 
assistance in the generation of colloids for line scan and TEMEDX measurements.

\section{References}

1 S. Sun, C. B. Murray, D. Weller, L. Folks and A. Moser, Science, 2000, 287, 1989-1992.

2 A. R. Wilson, K. Sun, M. Chi, R. M. White, J. M. LeBeau, H. H. Lamb and B. J. Wiley, J. Phys. Chem. C, 2013, 117, 17557-17566.

3 Y.-H. Chen and C.-S. Yeh, Chem. Commun., 2001, 371-372.

4 M. Hirano, K. Enokida, K. Okazaki, S. Kuwabata, H. Yoshida and T. Torimoto, Phys. Chem. Chem. Phys., 2013, 15, 7286-7294.

5 N. Toshima, T. Yonezawa and K. Kushihashi, J. Chem. Soc., Faraday Trans., 1993, 89, 2537-2543.

6 S. Link, Z. L. Wang and M. A. El-Sayed, J. Phys. Chem. B, 1999, 103, 3529-3533.

7 L. M. Liz-Marzan, Langmuir, 2006, 22, 32-41.

8 M. J. Beliatis, S. J. Henley, S. Ravi and P. Silva, Opt. Lett., 2011, 36(8), 1362-1364.

9 S. J. Henley, M. J. Beliatis, V. Stolojan, S. Ravi and P. Silva, Nanoscale, 2013, 5, 1054-1059.

10 S. Senapati, A. Ahmad, M. I. Khan, M. Sastry and R. Kumar, Small, 2005, 1, 517-520.

11 G. C. Papavassiliou, J. Phys. F: Met. Phys., 1976, 6, L103-L105.

12 A. Alqudami, S. Annapoorni, Govind and S. M. Shivaprasad, J. Nanopart. Res., 2008, 10, 1027-1036.

13 J. F. Sanchez-Ramirez, U. Pal, L. Nolasco-Hernandez, J. Mendoza-Alvarez and J. A. Pescador-Rojas, J. Nanopart. Res., 2008, 2008, 1-9.

14 K. Kim, K. L. Kim, J.-Y. Choi, H. B. Lee and K. S. Shin, J. Phys. Chem. C, 2010, 114, 3448-3453.

15 I. Lee, S. W. Han and K. Kim, Chem. Commun., 2001, 1782-1783. 16 J. Zhang, D. N. Oko, S. Garbarino, R. Imbeault, M. Chaker, A. C. Tavares, D. Guay and D. Ma, J. Phys. Chem. C, 2012, 116, 13413-13420.

17 S. Besner and M. Meunier, J. Phys. Chem. C, 2010, 114, 10403-10409.

18 D. Rioux, S. Vallières, S. Besner, P. Munoz, E. Mazur and M. Meunier, Adv. Opt. Mater., 2014, 2, 176-182.

19 N. G. Khlebtsov and L. A. Dykman, J. Quant. Spectrosc. Radiat. Transfer, 2010, 111, 1-35.

20 Y. Wang and Y. Ni, Talanta, 2014, 119, 320-330.

21 J. Deka, A. Paul and A. Chattopadhyay, J. Phys. Chem. C, 2009, 113, 6936-6947.

22 R. Intartaglia, G. Das, K. Bagga, A. Gopalakrishnan, A. Genovese, M. Povia, E. Di Fabrizio, R. Cingolani, A. Diasproa and F. Brandi, Phys. Chem. Chem. Phys., 2013, 15, 3075-3082.

23 A. Menéndez-Manjón, A. Schwenke, T. Steinke, M. Meyer, U. Giese, P. Wagener and S. Barcikowski, Appl. Phys. A: Mater. Sci. Process., 2013, 110, 343-350.

24 E. N. Sowa-Söhle, A. Schwenke, P. Wagener, A. Weiss, H. Wiegel, C. L. Sajti, A. Haverich, S. Barcikowski and A. Loos, Bionanomaterials, 2013, 14(3-4), 217-227.
25 P. Wagener, A. Schwenke and S. Barcikowski, Langmuir, 2012, 28, 6132-6140.

26 A. M. Dallaire, D. Rioux, A. Rachkov, S. Patskovsky and M. Meunier, J. Phys. Chem. C, 2012, 116, 11370-11377.

27 T. Li, B. Albee, M. Alemayehu, R. Diaz, L. Ingham, S. Kamal, M. Rodriguez and S. W. Bishnoi, Anal. Bioanal. Chem., 2010, 398(2), 689-700.

28 D. Mahl, J. Diendorf, S. Ristig, C. Greulich, Z. A. Li, M. Farle, M. Koller and M. Epple, J. Nanopart. Res., 2012, 14(10), 13.

29 S. Ibrahimkutty, P. Wagener, A. Menzel, A. Plech and S. Barcikowski, Appl. Phys. Lett., 2012, 101, 103104.

30 P. Wagener, S. Ibrahimkutty, A. Menzel, A. Plech and S. Barcikowski, Phys. Chem. Chem. Phys., 2013, 15, 3068-3074.

31 G. Compagnini, E. Messina, O. Puglisi, R. S. Cataliotti and V. Nicolosi, Chem. Phys. Lett., 2008, 457, 386-390.

32 D. Tiedemann, U. Taylor, C. Rehbock, J. Jakobi, S. Klein, W. A. Kues, S. Barcikowski and D. Rath, Analyst, 2014, 139, 931-942.

33 Z. Peng, B. Spliethoff, B. Tesche, T. Walther and K. Kleinermanns, J. Phys. Chem. B, 2006, 110, 2549-2554.

34 F. Calvo, Nanoalloys. From Fundamentals to Emergent Applications, Elsevier, 2013, pp. 39-66.

35 U. Kreibig and M. Vollmer, Optical Properties of Metal Clusters, Springer, Berlin, 1995.

36 A. Šileikaitè, J. Puišo, I. Prosyčevas and S. Tamulevičius, Mater. Sci.-Medzg., 2009, 15(1), 21-27.

37 K. G. Stamplecoskie and J. C. Scaiano, J. Am. Chem. Soc., 2010, 132, 1825-1827.

38 S. Link and M. A. El-Sayed, J. Phys. Chem. B, 1999, 103, 4212-4217.

39 W. Haiss, N. T. K. Thanh, J. Aveyard and D. G. Fernig, Anal. Chem., 2007, 79, 4215-4221.

40 M. Gaudry, J. Lerme, E. Cottancin, M. Pellarin, J. L. Vialle, M. Broyer, B. Prevel, M. Treilleux and P. Melinon, Phys. Rev. B: Condens. Matter Mater. Phys., 2001, 64, 085407.

41 K. D. Malviya and K. Chattopadhyay, J. Phys. Chem. C, 2014, 118, 13228-13237.

42 A. Hahn, G. Brandes, P. Wagener and S. Barcikowski, J. Controlled Release, 2011, 154, 164-170.

43 R. D. Glover, J. M. Miller and J. E. Hutchison, ACS Nano, 2011, 5, 8950-8957.

44 S. Grade, J. Eberhard, J. Jakobi, A. Winkel, M. Stiesch and S. Barcikowski, Gold Bull., 2013, 47, 83-93.

45 S. Kittler, C. Greulich, J. Diendorf, M. Köller and M. Epple, Chem. Mater., 2010, 22, 4548-4554.

46 N. Haustrup and G. M. O'Connor, Appl. Surf. Sci., 2013, 278, 86-91.

47 S. Dengler, C. Kübel, A. Schwenke, G. Ritt and B. Eberle, J. Opt., 2012, 14, 075203.

48 D. N. Oko, J. Zhang, S. Garbarino, M. Chaker, D. Ma, A. C. Tavares and D. Guay, J. Power Sources, 2014, 248, 273-282. 